\title{
CERTAIN INEQUALITIES CONNECTED WITH THE GOLDEN RATIO AND THE FIBONACCI NUMBERS
}

\author{
Marcin Adam, Bożena Piątek, Mariusz Pleszczyński, Barbara Smoleñ *, Roman Wituła \\ Institute of Mathematics, Silesian University of Technology \\ Gliwice, Poland \\ marcin.adam@polsl.pl,bozena.piatek@polsl.pl,mariusz.pleszczynski@polsl.pl \\ roman.witula@polsl.pl
}

\begin{abstract}
In the present paper we give some condensation type inequalities connected with Fibonacci numbers. Certain analytic type inequalities related to the golden ratio are also presented. All results are new and seem to be an original and attractive subject also for future research.
\end{abstract}

Keywords: Fibonacci numbers, golden ratio, Perrin constant

\section{Introduction}

The notion of Fibonacci numbers and the golden ratio may be found in many branches of mathematics, including number theory, geometry, algebra, matrix theory, numerical methods, classical analysis, dynamical systems, and even spectral analysis or music (see monographs [1-3], and selective papers [4-7]). Despite such a large spread occurrence of Fibonacci numbers and the golden ratio in mathematics, still some areas of mathematics tend to be poorly represented by these objects. In our opinion, a good example of such a niche (considered also in this paper) is the area of analytic inequalities. We believe this paper opens up a new stage of discoveries, and the inequalities presented here will be classical ones in the considered area of mathematics.

The main results of the paper are presented in two sections. In the first one we investigated the condensation type inequalities associated with the Fibonacci numbers. In the second one we discuss several analytic type inequalities related to the golden ratio and Perrin constant.

\footnotetext{
* Currently, the fourth author, Barbara Smolen, is a student of mathematics and this paper is a part of her Bachelor's thesis written under the supervision of Prof. Roman Wituła.
} 


\section{Condensation type inequalities connected with Fibonacci numbers}

We begin with the following inequality based on basic properties of the Fibonacci numbers. Let us recall that the Fibonacci numbers are defined by the following linear recurrence relation

$$
F_{n}=F_{n-1}+F_{n-2}, \quad n \in \mathbb{Z},
$$

with $F_{1}=F_{2}=1$. As a result of the definition we get $F_{0}=0$.

Theorem 1. Let $0<a_{1} \leq a_{2} \leq \ldots \leq a_{n+2}, n \in \mathbb{N}$ and $n \geq 2$ be a finite sequence of real numbers such that two inequalities are satisfied:

1.

$$
a_{1}+a_{2} \geq a_{n+2}
$$

2.

$$
F_{n+2} \geq 2^{k} \text { for some } k \in \mathbb{N} \text {. }
$$

Then there is an index $j \in\{1,2, \ldots, n\}$ such that

$$
a_{j}^{k}+a_{j+1}^{k}>a_{j+2}^{k}
$$

Proof. We prove this by contradiction. Let us suppose that for each index $j \in$ $\{1,2, \ldots, \mathrm{n}\}$ we have

$$
a_{j}^{k}+a_{j+1}^{k} \leq a_{j+2}^{k}
$$

which immediately leads to the following inequality

$$
a_{n+2}^{k} \geq F_{n+1} a_{2}^{k}+F_{n} a_{1}^{k}
$$

that can be easily shown by induction. Indeed, from (3) we have

$$
\begin{gathered}
a_{2}^{k} \geq a_{2}^{k}+0 \cdot a_{1}^{k}=F_{1} a_{1}^{k}+F_{0} a_{0}^{k}, \\
a_{3}^{k} \geq a_{2}^{k}+a_{1}^{k}=F_{2} a_{2}^{k}+F_{1} a_{1}^{k},
\end{gathered}
$$

for the initial step and

$$
a_{i+2}^{k} \geq a_{i+1}^{k}+a_{i}^{k} \geq F_{i} a_{2}^{k}+F_{i-1} a_{1}^{k}+F_{i-1} a_{2}^{k}+F_{i-2} a_{1}^{k}=F_{i+1} a_{2}^{k}+F_{i} a_{1}^{k}
$$

for the inductive one.

From (4), on account of (1) we obtain

$$
\left(a_{1}+a_{2}\right)^{k} \geq F_{n+1} a_{2}^{k}+F_{n} a_{1}^{k} .
$$


Next, let us denote $a_{2}=a_{1}+\delta$, where $\delta>0$. Then the left-hand side of the previous inequality is equal to the following:

$$
\left(a_{1}+a_{2}\right)^{k}=2^{k} a_{1}^{k}+\sum_{i=1}^{k}\left(\begin{array}{c}
k \\
i
\end{array}\right) 2^{k-i} a_{1}^{k-i} \delta^{i} .
$$

Now from (2) it follows directly that $F_{n+1}>2^{k-i}$ for all $i \in\{1,2, \ldots, k\}$. Indeed, $F_{n+1}+F_{n} \geq 2 \cdot 2^{k-i}$ and since $n \geq 2$, there is $F_{n+1}>F_{n}$. So finally we obtain

$$
\left(a_{1}+a_{2}\right)^{k}<F_{n+2} a_{1}^{k}+\sum_{i=1}^{k} F_{n+1}\left(\begin{array}{c}
k \\
i
\end{array}\right) a_{1}^{k-i} \delta^{i}=F_{n} a_{1}^{k}+F_{n+1} a_{2}^{k},
$$

which contradicts to (4). This completes the proof.

As a direct conclusion of this result we obtain the following generalization:

Corollary 2. Let $0<a_{1} \leq a_{2} \leq \ldots \leq a_{n+2}, n \in \mathbb{N}$ and $n \geq 2$ be a finite sequence of real numbers satisfying condition 1 of the previous theorem. If, additionally,

$$
\left(a_{1}+a_{2}\right)^{x}<F_{n+1} a_{2}^{x}+F_{n} a_{1}^{x}
$$

for some $x>0$, then there is an index $j \in\{1,2, \ldots, n\}$ such that

$$
a_{j}^{x}+a_{j+1}^{x}>a_{j+2}^{x} .
$$

Remark 3. For the inequality (2) see also Chern and Cui paper [8].

\section{Inequalities connected with the golden ratio}

Let $\varphi$ denote the golden ratio, i.e. $\varphi=\frac{1+\sqrt{5}}{2} \approx 1.618034$ and let $\bar{\varphi}:=\frac{1-\sqrt{5}}{2} \approx$ $\approx-0.618034$.

Theorem 4. The following golden ratio type inequalities hold:

1.

$$
0.12>f(x):=\frac{\varphi(\varphi+1)}{x(x+1)}-e^{\varphi-x} \geq 0
$$

for $x>0$; the equality sign is attained for $x=\varphi$ only. The function $f$ is increasing on interval $\left[\varphi, x_{0}\right]$ and decreasing on each of the intervals $(0, \varphi)$ and $\left(x_{0}, \infty\right)$, where $x_{0} \approx 3.90023$ (see Fig. 1). We note that

$$
\max \{f(x): x>\varphi\}=f\left(x_{0}\right) \approx 0.119584 .
$$

2. The function

$$
g(x):=\frac{x+1}{\varphi+1}-e^{\frac{x-\varphi}{\varphi x}}
$$


is increasing on each of the intervals $\left(0, x_{1}\right)$ and $(\varphi, \infty)$, and decreasing on interval $\left(x_{1}, \varphi\right)$, where $x_{1} \approx 0.214714$. We note that $g(\varphi)=0$ and

$$
\max \{g(x): 0<x<\varphi\}=g\left(x_{1}\right) \approx 0.44637 .
$$

3. The function

$$
h_{c}(x):=x-\sqrt{\varphi}-\arccos \frac{1}{x}+\arccos \frac{1}{\sqrt{\varphi}}
$$

is decreasing on $(1, \sqrt{\varphi})$ and increasing on $(\sqrt{\varphi}, \infty)$.

Moreover, we have $h_{c}(\sqrt{\varphi})=0$.

4. Let us set

$$
F(x):=\ln \frac{x+2}{\varphi+2}-\arctan \frac{x-\varphi}{\varphi x+1},
$$

for $x \in(-2, \bar{\varphi}) \cup(\bar{\varphi}, \infty)$, and

$$
G(x):=\ln \frac{x+2}{\bar{\varphi}+2}-\arctan \frac{x-\bar{\varphi}}{\bar{\varphi} x+1},
$$

for $x \in(-2, \varphi) \cup(\varphi, \infty)$. Then the function $F(x)$ is increasing on each of intervals $(-2, \bar{\varphi})$ and $(\varphi, \infty)$, and decreasing on interval $(\bar{\varphi}, \varphi)$. On the other hand, the function $G(x)$ is increasing on each of the intervals $(-2, \bar{\varphi})$ and $(\varphi, \infty)$, and decreasing on $(\bar{\varphi}, \varphi)$. Furthermore we obtain

$$
F(\varphi)=G(\bar{\varphi})=0,
$$

$$
\begin{gathered}
\lim _{x \rightarrow \bar{\varphi}_{-}} F(x)=\ln \frac{\bar{\varphi}+2}{\varphi+2}-\frac{\pi}{2}=\ln \left(\frac{1}{2}(3-\sqrt{5})\right)-\frac{\pi}{2} \approx-2.53322, \\
\lim _{x \rightarrow \bar{\varphi}_{+}} F(x)=\ln \frac{\bar{\varphi}+2}{\varphi+2}+\frac{\pi}{2}=\pi-2.53322 \approx 0.608373 \\
\frac{\bar{\varphi}+2}{\varphi+2}=\frac{\sqrt{5}}{3 \varphi+1},
\end{gathered}
$$

$$
\lim _{x \rightarrow \varphi_{-}} G(x)=\ln \frac{\varphi+2}{\bar{\varphi}+2}-\frac{\pi}{2}=\ln \left(\frac{1}{2}(3+\sqrt{5})\right)-\frac{\pi}{2} \approx 2.53322 \text {, }
$$

$\lim _{x \rightarrow \varphi_{+}} G(x)=\ln \frac{\varphi+2}{\bar{\varphi}+2}+\frac{\pi}{2}=\ln \left(\frac{1}{2}(3+\sqrt{5})\right)+\frac{\pi}{2} \approx-\pi+2.53322 \approx-0.608373$.

Moreover, if $x \in[0, \varphi-\bar{\varphi}]=[0, \sqrt{5}]$ then (see Figs. 2-4) 


$$
\begin{gathered}
F(\bar{\varphi}+x)+G(\varphi-x)= \\
=\ln \left(5+\sqrt{5} x-x^{2}\right)-\ln 5-\arctan \left(\varphi \frac{\sqrt{5}-x}{x}\right)-\arctan \left(\bar{\varphi} \frac{\sqrt{5}-x}{x}\right)= \\
=\ln \left(5+\sqrt{5} x-x^{2}\right)-\ln 5-\arctan \left(\frac{x(\sqrt{5}-x)}{x^{2}+(\sqrt{5}-x)^{2}}\right)
\end{gathered}
$$

and the minimum of this function is attained in $x=\frac{\varphi-\bar{\varphi}}{2}=\frac{\sqrt{5}}{2}$, we have

$$
F\left(\frac{1}{2}\right)+G\left(\frac{1}{2}\right)=\ln \frac{5}{4}-\arctan \frac{1}{2} \approx-0.240504057
$$

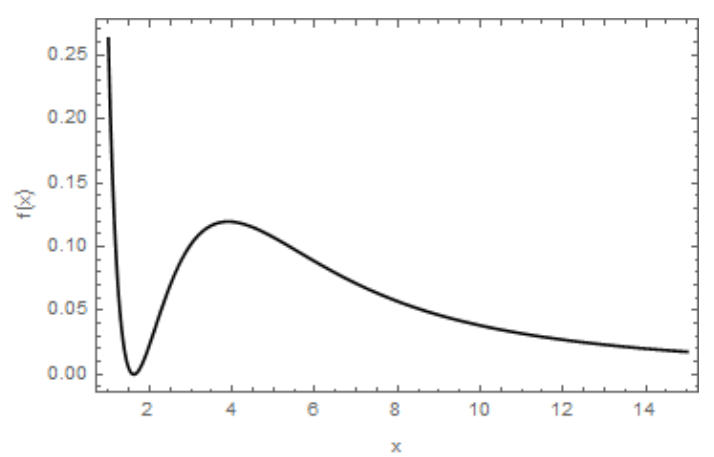

Fig. 1. Plot of the function $f(x)$

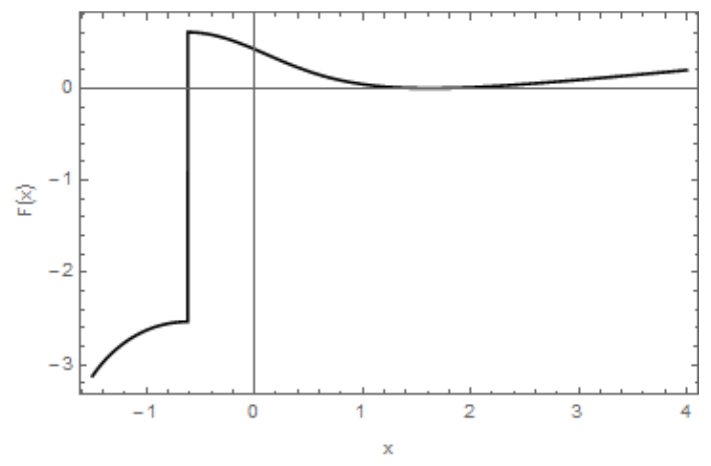

Fig. 2. Plot of the function $F(x)$ 


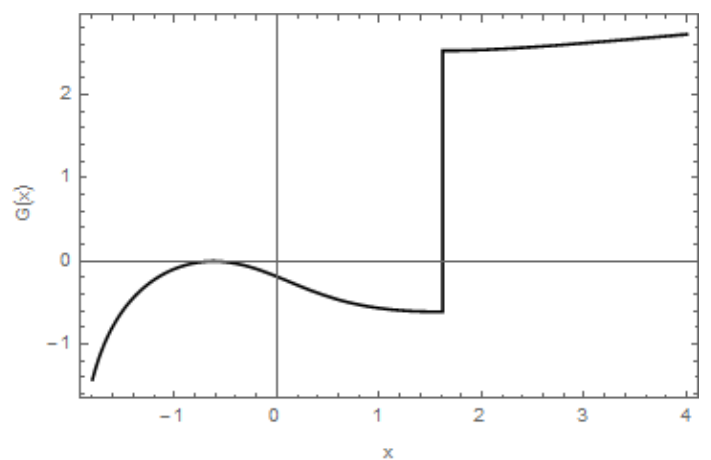

Fig. 3. Plot of the function $G(x)$

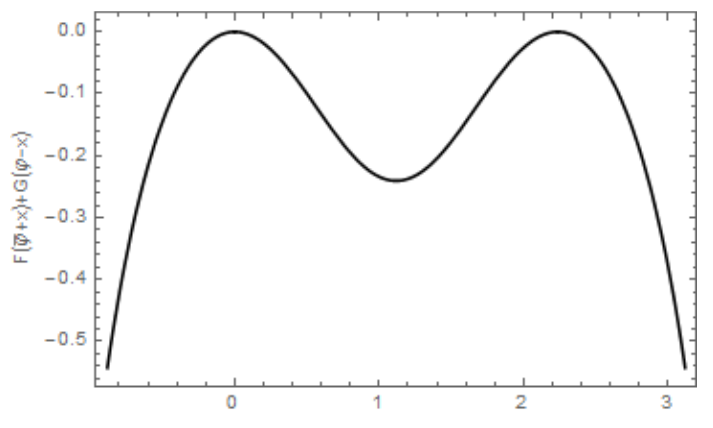

Fig. 4. Plot of the function $F(\bar{\varphi}+x)+G(\varphi-x)$ in the interval $\left[\frac{\sqrt{5}}{2}-2, \frac{\sqrt{5}}{2}+2\right]$ (the domain of this one is equal to $(\sqrt{5} \bar{\varphi}, \sqrt{5} \varphi)$ )

Proof. We consider the following functions:

$$
\begin{gathered}
f_{1}(x):=x-\ln \left(x^{2}+x\right), \quad x \in(0, \infty), \\
g_{1}(x):=(x+1) e^{\frac{1}{x}}, x \in(0, \infty), \\
F_{1}(x):=\ln (x+2)+\arctan \frac{1}{x}, x \in(0, \infty) .
\end{gathered}
$$

Computing derivatives of these functions we obtain

$$
\begin{gathered}
f_{1}^{\prime}(x)=\frac{x^{2}-x-1}{x^{2}+x}=\frac{(x-\bar{\varphi})(x-\varphi)}{x(x+1)}, \\
g_{1}^{\prime}(x)=\frac{x^{2}-x-1}{x^{2}} e^{\frac{1}{x}}=\frac{(x-\bar{\varphi})(x-\varphi)}{x^{2}} e^{\frac{1}{x}} \\
F_{1}^{\prime}(x)=\frac{x^{2}-x-1}{(x+2)\left(x^{2}+1\right)}=\frac{(x-\bar{\varphi})(x-\varphi)}{(x+2)\left(x^{2}+1\right)}
\end{gathered}
$$


Moreover,

$$
h_{c}^{\prime}(x)=\frac{x^{4}-x^{2}-1}{\left(x \sqrt{x^{2}-1}\right)\left(x \sqrt{x^{2}-1}+1\right)}=\frac{\left(x^{2}-\bar{\varphi}\right)(x-\sqrt{\varphi})(x+\sqrt{\varphi})}{\left(x \sqrt{x^{2}-1}\right)\left(x \sqrt{x^{2}-1}+1\right)} .
$$

It is easy to check that the function $f_{1}(x)$ is decreasing on $(0, \varphi)$ and is increasing on $(\varphi, \infty)$, so we have $f_{1}(x) \geq f_{1}(\varphi)$ for $x>0$ which is equivalent to the inequality $f(x) \geq 0$ for $x>0$ (the equality sign is attained here only for $x=\varphi$ ). By numerical calculations, we proved that the function $f(x)$ is increasing on interval $\left[\varphi, x_{0}\right]$ and decreasing on intervals $(0, \varphi)$ and $\left(x_{0}, \infty\right)$, where $x_{0} \approx 3.90023$.

Similarly as $f_{1}(x)$, also $g_{1}(x)$ is decreasing on $(0, \varphi)$ and is increasing on $(\varphi, \infty)$, so we obtain

$$
(x+1) e^{\frac{1}{x}} \geq(\varphi+1) e^{\frac{1}{\varphi}}, \quad x>0,
$$

i.e.

$$
\frac{x+1}{\varphi+1} \geq e^{\frac{x-\varphi}{\varphi x}}
$$

We have $g^{\prime}(x)=\frac{1}{\varphi^{2}}-\frac{1}{x^{2}} e^{\frac{x-\varphi}{\varphi x}}$. By numerical calculations we proved that the function $g(x)$ is increasing on intervals $\left(0, x_{1}\right)$ and $(\varphi, \infty)$, and decreasing on interval $\left(x_{1}, \varphi\right)$. The function $h_{c}(x)$ is decreasing on $(1, \sqrt{\varphi})$ and increasing on $(\sqrt{\varphi}, \infty)$. Hence, function $h_{c}(x)$ has a local minimum at the point $\sqrt{\varphi}$ which is equal to $h_{c}(\sqrt{\varphi})=0$.

Corollary 5. By item 1, the following inequality holds

$$
1+\frac{(\varphi-x)(x+1+\varphi)}{x(x+1)} \geq e^{\varphi-x}, \quad x>0 .
$$

In equivalent form, we obtain

$$
\ln \left(1+z-(2 \bar{\varphi}+1) z^{2}\right) \leq z, \quad z>-\varphi .
$$

Proof. We have

$$
x-\ln \left(x^{2}+x\right) \geq \varphi-\ln \left(\varphi^{2}+\varphi\right),
$$

i.e.

$$
x-\varphi \geq \ln \left(1+\frac{(x-\varphi)^{2}+(2 \varphi+1)(x-\varphi)}{2 \varphi+1}\right)
$$

which implies (5) for $z:=x-\varphi$ since $\varphi^{2}=\varphi+1$ and $\frac{1}{2 \varphi+1}=-(2 \bar{\varphi}+1)$. 
Corollary 6. By item 2, the following inequality holds

$$
1-t \geq e^{\frac{t}{1-\varphi t}}, \quad t<\frac{1}{\varphi} .
$$

Corollary 7. By item 3, the following inequality holds

$$
x-\sqrt{\varphi} \geq \arcsin \frac{1}{\sqrt{\varphi}}-\arcsin \frac{1}{x}, \quad x \geq .1
$$

More precisely, the function

$$
h_{s}(x):=x-\sqrt{\varphi}-\arcsin \frac{1}{\sqrt{\varphi}}+\arcsin \frac{1}{x}
$$

is decreasing on interval $(1, \sqrt{\varphi})$ and increasing on interval $(\sqrt{\varphi}, \infty)$.

Remark 8. Closely connected to the golden ratio is the so-called Perrin constant $\lambda_{0}$ defined to be the only real zero of the so-called Perrin polynomial (see [9-11])

$$
P(x):=x^{3}-x-1=\left(x-\lambda_{0}\right)\left(x-\frac{i}{\sqrt{\lambda_{0}}} e^{i \Psi}\right)\left(x+\frac{i}{\sqrt{\lambda_{0}}} e^{-i \Psi}\right)
$$

where

$$
\begin{gathered}
\Psi:=\arcsin \left(\frac{1}{2} \sqrt{\lambda_{0}^{3}}\right), \quad \sqrt[3]{18} \lambda_{0}=\sqrt[3]{9+\sqrt{69}}+\sqrt[3]{9-\sqrt{69}} \\
\frac{i}{\sqrt{\lambda_{0}}} e^{i \Psi}=\frac{1}{2 \sqrt[3]{18}}(-\sqrt[3]{9+\sqrt{69}}-\sqrt[3]{9-\sqrt{69}}+i \sqrt{3}(\sqrt[3]{9+\sqrt{69}}-\sqrt[3]{9-\sqrt{69}})),
\end{gathered}
$$

and $\lambda_{0} \approx 1.324717957244746$.

In relation to inequalities

$$
F(x) \geq F(\varphi)=0, \quad x \geq \varphi,
$$

and

$$
F(x) \leq F(\bar{\varphi}), \quad x \in(-2, \bar{\varphi})
$$

from point 4 of Theorem 4 , we are interested in the equivalent of these inequalities for the Perrin constant $\lambda_{0}$, i.e. the inequalities of the type

$$
r(x)\left\{\begin{array}{l}
\leq \\
\geq
\end{array}\right\} r\left(\lambda_{0}\right), x \in(\alpha, \beta), \lambda_{0} \in(\alpha, \beta),
$$


where

$$
r(x)=r(x ; a, b, c)=\ln ( \pm x(x+a))-\arctan \frac{b}{x+c} .
$$

Since we have

$$
r^{\prime}(x)=\frac{2 x^{3}+(a+b+4 c) x^{2}+\left(2 a c+a b+2\left(b^{2}+c^{2}\right)\right) x+a\left(b^{2}+c^{2}\right)}{x(x+a)\left((x+c)^{2}+b^{2}\right)}
$$

so we are interested when the following system of equations hold:

$$
\left\{\begin{array}{c}
a+b+4 c=0, \\
2 a c+a b+2\left(b^{2}+c^{2}\right) \\
a\left(b^{2}+c^{2}\right)=-2,
\end{array}=-2, \Leftrightarrow\left\{\begin{array}{c}
a=-b-4 c, \\
b^{2}-6 b c-6 c^{2}=-2, \\
b^{3}+4 c b^{2}+b c^{2}+4 c^{3}=2,
\end{array}\right.\right.
$$

which implies that $b$ is a real solution of the following equation

$$
-368-720 x+516 x^{2}-1440 x^{3}+1836 x^{4}+1445 x^{6}=0
$$

i.e.

$$
b \approx-0.3293532687 \text { or } b \approx 0.84778730534 .
$$

Finally, by numerical calculations we get precisely two triplets of real numbers being the solution of system (6):

1) $a \approx-2.790347073, b \approx-0.3293532687, c \approx 0.779925085$,

2) $a \approx-2.334186207, b \approx 0.8477873053, \quad c \approx 0.371599725$.

For these solutions we can deduce the following inequalities:

A) the first collection of five inequalities for the first triple $(a, b, c)$ of solutions (see Fig. 5)

$$
\Lambda(x)>0 \text { for } x \in(0.298336 \ldots, 2.41697 \ldots),
$$

where

$$
\Lambda(x):=\ln (-x(x+a))-\arctan \frac{b}{x+c}, \quad x \in(0,-a),
$$

and

$$
\begin{gathered}
\Lambda(x)<0 \text { for } x \in(0,0.298336 \ldots) \cup(2.41697 \ldots,-a), \\
\Lambda(x)>x-\lambda_{0} \text { for } x \in(0.0727841 \ldots, 1.94339 \ldots), \\
\Lambda(x)<x-\lambda_{0} \text { for } x \in(0,0.0727841 \ldots) \cup(1.94339 \ldots,-a), \\
\ln \left(\frac{x(x+a)}{\lambda_{0}\left(\lambda_{0}+a\right)}\right) \leq \arctan \frac{b}{x+c}-\arctan \frac{b}{\lambda_{0}+c}=\arctan \frac{b\left(\lambda_{0}-x\right)}{(x+c)\left(\lambda_{0}+c\right)+b^{2}},
\end{gathered}
$$


for $x \in(0,-a)$ and where the equality sign is taken only for $x=\lambda_{0}$.

The function

$$
\Lambda_{1}(x):=\ln \left(-\frac{x(x+a)}{\lambda_{0}\left(\lambda_{0}+a\right)}\right)-\arctan \frac{b\left(\lambda_{0}-x\right)}{(x+c)\left(\lambda_{0}+c\right)+b^{2}}
$$

is increasing on $(-a, \infty), \Lambda_{1}(x)=0 \Leftrightarrow x \approx 3.40586$. Moreover, $\Lambda_{1}(x)$ is decreasing on two intervals $\left(-\infty, x_{0}=-\frac{b^{2}}{\lambda_{0}+c}-c\right)$ and $\left(x_{0}, 0\right)$, and

$$
\begin{aligned}
& \lim _{x \rightarrow x_{0}^{-}} \Lambda_{1}(x)-\lim _{x \rightarrow x_{0}^{+}} \Lambda_{1}(x)=\pi, \\
& \Lambda_{1}(x)>0, \quad x \in\left(-\infty, x_{0}\right), \\
& \Lambda_{1}(x)<0, \quad x \in\left(x_{0}, 0\right) ;
\end{aligned}
$$

B) for the second triple $(a, b, c)$ of solutions similar inequalities can be obtained, however, due to the volume of the paper, they will be omitted.
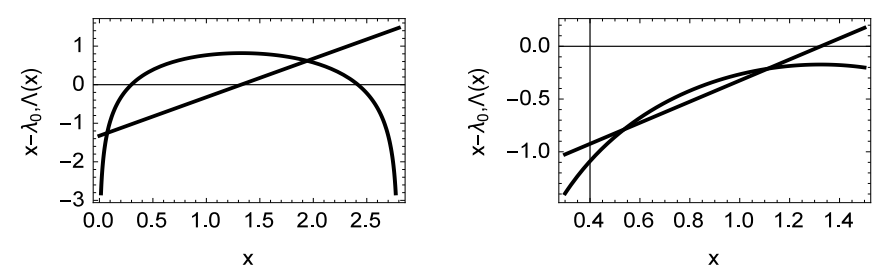

Fig. 5. Plot of the functions $x-\lambda_{0}$ and $\Lambda(x)$ for the first triple $(a, b, c)$ - on the left, and for the second triple $(a, b, c)$ - on the right

\section{Conclusions}

In the paper certain inequalities connected with the golden ratio and the Fibonacci numbers are discussed. We were able to accomplish the intended overall goal of the paper, even with some excess (see in particular the results of point 4 of Theorem 4). Generalization from Remark 8 connected with the Perrin's polynomial and constant is quite natural and in fact well-defined, but did not completely fulfill our expectations of aesthetic nature. We believe that the research subject matter indicated in this paper is still open and can encourage (especially Fibomaniacs) for active reflection. 


\section{References}

[1] Dunlop R., The Golden Ratio and Fibonacci Numbers, World Scientific, Singapore 2006.

[2] Vajda S., Fibonacci and Lucas Numbers, and the Golden Section Theory and Applications, Dover Publications Inc., New York 2008.

[3] Hoggatt V.E., Fibonacci and Lucas Numbers, The Fibonacci Association, Santa Clara 1979.

[4] Mongoven C., Sonification of multiple Fibonacci-related sequences, Annales Mathematicae et Informaticae 2013, 41, 175-192.

[5] Wituła R., Słota D., Hetmaniok E., Bridges between different known integer sequences, Annales Mathematicae et Informaticae 2013, 41, 255-263.

[6] Słanina P., Generalizations of Fibonacci polynomials and free linear groups, Linear and Multilinear Algebra, DOI: 10.1080/03081087.2015.1031073.

[7] Herz-Fischler R., A "very pleasant" theorem, College Mathematics Journal 1993, 24, 4, 318-324.

[8] Chern S., Cui A., Fibonacci numbers close to a power of 2, The Fibonacci Quarterly 2014, 52, 4, 344-348.

[9] Hetmaniok E., Wituła R., Lorenc P., Pleszczyński M., On an improvement of the numerical application for Cardano's formulae in Mathematica software (in review).

[10] Wituła R., Lorenc P., Różański M., Szweda M., Sums of the rational powers of roots of cubic polynomials, Zeszyty Naukowe Politechniki Śląskiej, Seria Matematyka Stosowana 2014, 4, 17-34.

[11] Dubickas A., Hare K.G., Jankauskas J., There are no two nonreal conjugates of a Pisot number with the same imaginary part, arXiv:1410.1600v1 [math.NT]. 